TITLE:

\title{
Spiral structures in turbulent flow
}

$\operatorname{AUTHOR}(S):$

Moffatt, H.K.

CITATION:

Moffatt, H.K.. Spiral structures in turbulent flow. 数理解析研究所講究録 1993, 852: 104-111

ISSUE DATE:

1993-10

URL:

http://hdl.handle.net/2433/83718

RIGHT: 


\title{
Spiral structures in turbulent flow
}

\author{
By H.K. Moffatt \\ Department of Applied Mathematics and Theoretical Physics, \\ Silver Street, Cambridge, CB3 9EW.
}

\section{Introduction}

Spiral structures are natural candidates for the role of the 'generic structures' of turbulent flow, because they are the eventual outcome of KelvinHelmholtz instability, an all-pervasive phenomenon associated with nearly all shear flows at very high Reynolds number. Such structures were proposed by Lundgren (1982) in a model of the fine structure of turbulence in which axial stretching of rolled-up spiral vortices played an essential role. This model could be viewed as a natural development of Townsend's (1951) model of the dissipative structures of turbulence in terms of a random distribution of vortex sheets and/or tubes, each such structure being subjected to the local rate of strain (assumed uniform and constant) associated with all other vortex structures (see Batchelor 1982, §7.4). It is known that compressive strain (with two positive principle rates of strain) is more likely in isotropic turbulence than extensive strain, so that sheets form with higher probability than tubes. However these are immediately subject to the Kelvin-Helmholtz instability which may be impeded, but not entirely suppressed, by the stretching process.

A random superposition of vortex sheets has the property that the resulting velocity field has a finite number of discontinuities per unit length on any straight line transversal. This leads to an energy spectrum function $E(k)$ proportional to $k^{-2}$ (with an exponential cut-off at wave-numbers of the order of the reciprocal of the typical vortex sheet thickness). The Kolmogorov cascade theory, by contrast, gives $E(k)$ proportional to $k^{-5 / 3}$ (with an exponential cut-off at the inner Kolmogorov scale). It is natural then to enquire as to what structures in $x$-space can give rise to such a power-law. This question was raised by Moffatt (1984) where it was argued that the accumulation points of discontinuities associated with spiral structures could give rise to fractional power laws $k^{-\lambda}$ with $1<\lambda<2$. We shall pursue this argument a little further in the present note.

The influence of spiral structures in two-dimensional turbulence has been considered by Gilbert (1988) who considered the wind-up of a weak

\footnotetext{
This paper is to apfear in the Proceedings of the IMA Conference "Fractals, Wavelets and Fourier Transforms: new developments and new afflications". (Ed. M.Farge er al) Clarencion (1993), and is reprinted here with permission.
} 


\section{Spiral structures in turbulent flow}

vortex patch by a strong concentrated vortex in its vicinity, and showed that fractional power laws $E(k) \sim k^{-\lambda}$ with $3<\lambda<4$ could be obtained through this mechanism. This provides a convenient bridge (and reconciliation) between the vortex patch theory of Saffman (1971) and the enstrophy cascade theory of Kraichnan (1967) and Batchelor (1969) which give $k^{-4}$ and $k^{-3}$ respectively. The methods that we adopt in the present paper are similar to those employed by Gilbert.

\section{The Kelvin-Helmholtz instability}

A vortex sheet, i.e. a tangential discontinuity (of magnitude $U$ ) in tangential velocity, is well known to be unstable, on linear analysis, to perturbations of wavenumber $k$, the growth rate being of order $k U$. The fact that the shortest wavelengths grow most rapidly means that the linear problem is ill-posed, and, as shown by Moore (1979), the nonlinear development of an initially sinusoidal perturbation develops a mild singularity at a finite time $t_{c}$ of order $(k U)^{-1}$, largely as a result of the singular character of the dispersion relation. The mechanism of the instability, as described by Batchelor (1967), is that the strength of the vortex sheet increases near the inflexion points where the surface is tilted in the sense favoured by the velocity difference; this tendency persists in the nonlinear regime, and at time $t=t_{c}$, the strength of the vortex sheet is cusp-shaped at the inflexion point (see figure 1).

Numerical procedures based on the Euler equations cannot be continued beyond this finite time singularity. If however the equations governing the evolution of the sheet are modified slightly, in a manner that mimics the fact that any vortex sheet in a real fluid would not be a perfect discontinuity, but would have finite thickness, then the integration can be pursued beyond $t=t_{c}$. Such a 'desingularisation' procedure has been adopted by Krasny (1986) who integrated the equations for vortex sheet evolution for various small values of a desingularisation parameter $\delta$. When $\delta=0$, the evolution based on the Euler equations is recovered. Figure 2 shows a reproduction of Krasny's solution for the vortex sheet, for $\delta=0.05$, and for $t=8 t_{c} / 3$. At this stage, five complete turns have appeared in the rolled-up vortex sheet. As $\delta$ increases, the number of turns (at fixed $t$ ) decreases; conversely, it may be supposed that, as $\delta$ decreases towards 0 , the number of turns at fixed $t\left(>t_{c}\right)$ increases without limit.

A straight line transversal passing through the centre of this spiral now samples an accumulation point of discontinuities of the velocity field. It is this property that can lead to a fractional power law in the energy spectrum. A random distribution of spiral vortices of this kind can therefore, in principle, yield a Kolmogorov spectrum (or any other power law spectrum) if the structure is right. We investigate this by an even more simplified 


\section{By H.K. Moffatt}

(a)

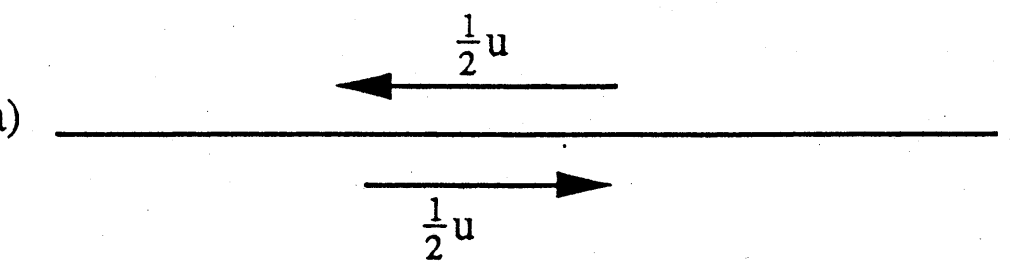

(b)

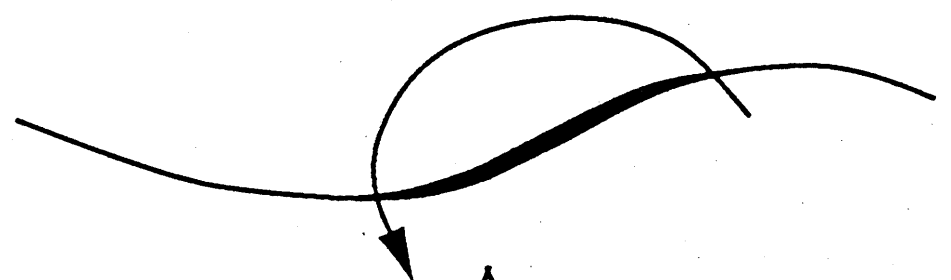

(c)

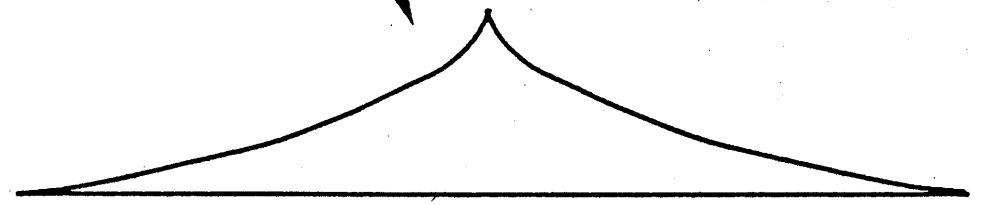

(d)

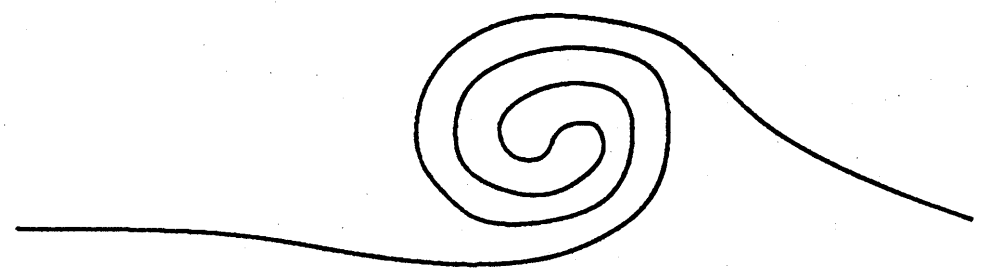

Figure 1. The mechanism of Kelvin-Helmholtz instability. (a) The unperturbed state; (b) the instability mechanism associated with accumulation of vorticity at the inflexion point; (c) vortex sheet strength, showing the cusp at the inflexion point at the instant $t=t_{c}$ when the curvature becomes discontinuous (Moore 1979); (d) development of the spiral singularity for $t>t_{c}$.

model in the following section.

\section{A simple model}

Consider a set of concentric circular vortex sheets of radius $x_{m}=\pi m^{-\alpha}$ $(m=1,2,3$ etc. $)$. Let $\delta x_{m}=x_{m}-x_{m+1}\left(\sim m^{-\alpha+1}\right.$ for large $\left.m\right)$, and suppose that the velocity in the annulus $\delta x_{m}$ is $u_{m} \sim m^{\beta} \sim x_{m}^{-\frac{\beta}{\alpha}}$. The energy in the annulus $\delta x_{m}$ is then $E_{m} \sim \int_{x_{m+1}}^{x_{m}} u_{m}^{2} x_{m} d x_{m} \sim m^{2 \beta-2 \alpha-1}$, and the condition that the total energy be finite then implies that $\beta<\alpha$. The situation is shown schematically in figure 3.

The spectrum of the velocity field may be calculated by the method 


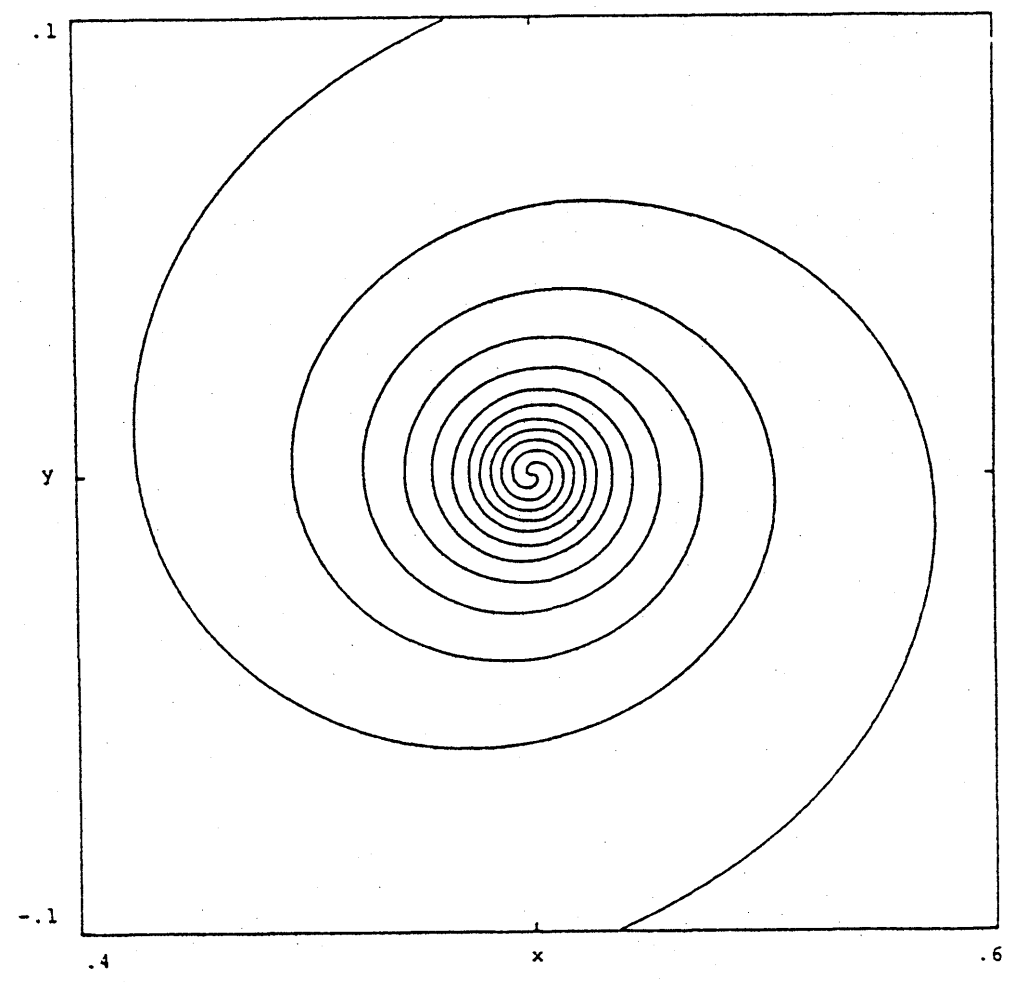

Figure 2. (From Krasny 1986) Structure of the spiral vortex computed by Krasny according to a desingularised prescription.

developed by Gilbert (1988). The Fourier coefficients of $u(x)$ are given by

$$
\begin{aligned}
a_{n} & =\frac{2}{\pi} \int_{0}^{\pi} u(x) \sin n x d x \\
& =\frac{4}{n \pi} \sum_{m=1}^{\infty} u_{m} \sin \frac{1}{2} n\left(x_{m+1}+x_{m}\right) \sin \frac{1}{2} n\left(x_{m+1}-x_{m}\right) .
\end{aligned}
$$

The factor $\sin \left(\frac{1}{2} n \delta x_{m+1}\right)$ is effectively zero if $m^{(\alpha+1)} \gg n$; hence, provided $u_{m}$ does not increase too rapidly with $m,{ }^{1}$ the number of terms making significant contributions to this sum is $M=O\left(n^{\frac{1}{\alpha+1}}\right)$. The term $R_{m}=$ $\sin \frac{1}{2} n\left(x_{m+1}+x_{m}\right)$ is effectively a random number in the interval $[-1,1]$, and the sum can be thought of as the result of a random walk in which the step-length increases like $m^{\beta}$ (where $m$ labels the step). It is not difficult

1 J.C. Vassilicos (private communication) has calculated that the condition for validity of this argument is $\beta \leq \frac{1}{2} \alpha$. 


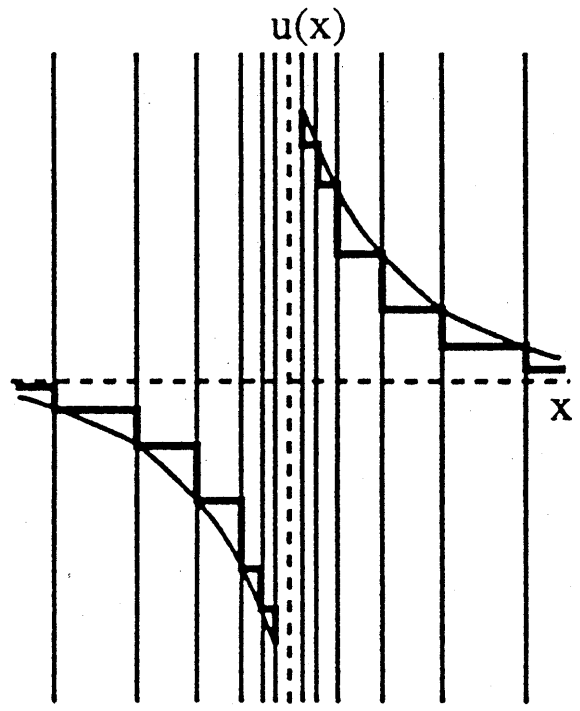

(b)

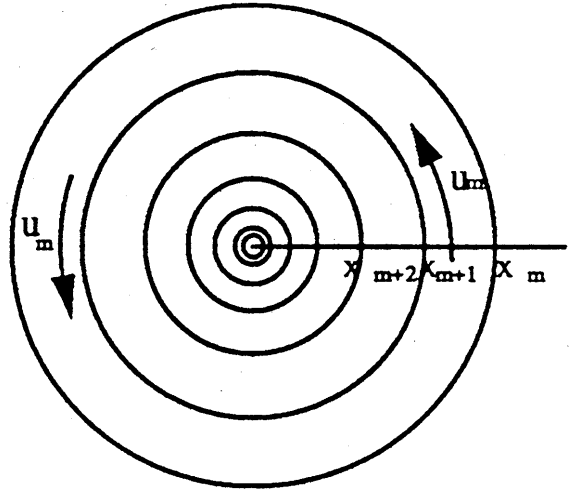

(a)

Figure 3. (a) Simplified model involving concentric vortex sheets; (b) the corresponding velocity profile on a transversal through the centre.

to deduce that

$$
\left|a_{n}^{2}\right| \sim n^{-\lambda}, \text { where } \lambda=2-\frac{2 \beta+1}{\alpha+1} .
$$

If there is a random superposition of such structures, then a straight line transversal will not pass through the centre of all of them, but rather will pass at distance $y$ from the centre where all values of $y$ between -1 and +1 are sampled with equal probability. Clearly, when $y$ is small but non-zero, the infinite sum in the above equation is cut-off at a large but finite value of $m$. The resulting value of $a_{n}^{2}$ can then be averaged over all values of $y$, giving the modified result

$$
\left\langle a_{n}^{2}(y)\right\rangle \sim n^{-\mu} \quad \text { where } \quad \mu=\lambda+\frac{\alpha}{1+\alpha} .
$$

We may note here in parenthesis the special values $\beta=0, \alpha=\frac{1}{2}$, for which $\lambda=\frac{4}{3}, \mu=\frac{5}{3}$. These values were relevant in the work of Gilbert (1988), who considered however not the wind-up of a vortex sheet, but the wind-up of a discontinuity of vorticity (rather than of velocity) by a concentrated point-vortex. Consequently, in the simplest configuration considered, Gilbert found an enstrophy spectrum proportional to $k^{-\frac{5}{3}}$, and a corresponding energy spectrum proportional to $k^{-\frac{11}{3}}$. 


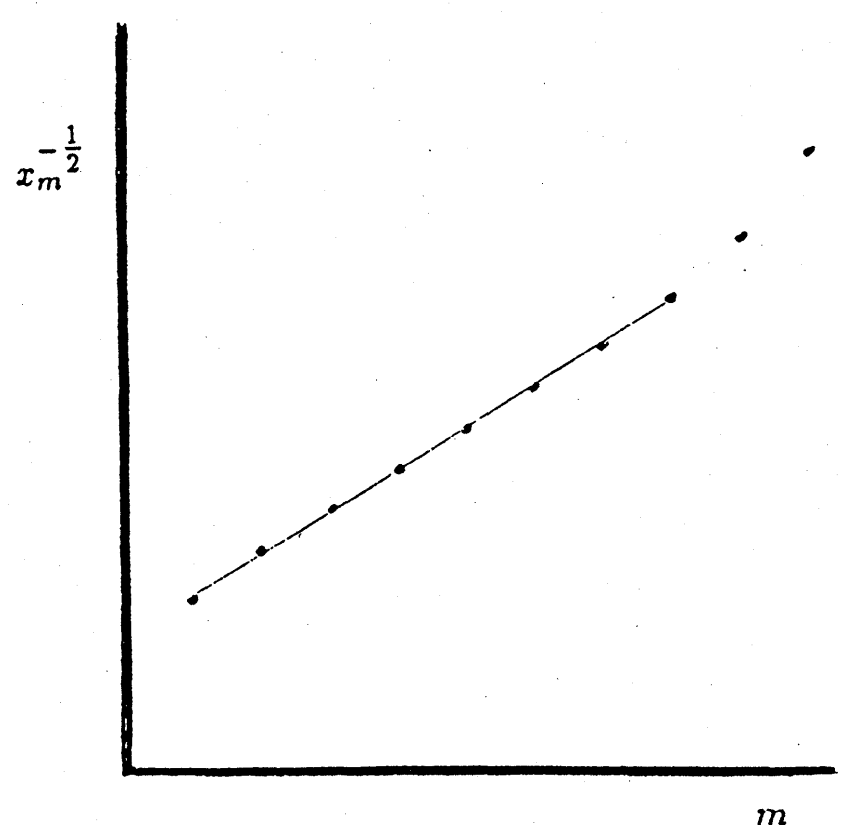

Figure 4. Plot of $x_{m}^{-\frac{1}{2}}$ against $m$ for the Krasny vortex showing a linear range for $1 \leqslant m \leqslant 8$. This implies that the spiral can be represented reasonably well by the curve $r\left(\theta-\theta_{0}\right)^{2}=$ cst., and that the circulating velocity behaves like $r^{-\frac{1}{2}}$, except in the very central region.

It is interesting to re-examine the structure of the Krasny vortex in relation to this analysis. Figure 4 shows a plot of $x_{m}^{-\frac{1}{2}}$, against $m$, where $m$ labels the intersections of the spiral with the $x$-axis, as before. There is a good straight-line relationship for $1 \leqslant m \leqslant 8$, consistent with fitting to a spiral of the form $r\left(\theta-\theta_{0}\right)^{2}=$ cst.. $^{2}$ The points for $m=9,10,11$, depart from this straight-line behaviour, and this is presumably due to the fact that the vortex does not achieve an asymptotic structure in the central region (or perhaps the desingularisation procedure is at its worst in this region).

With $\alpha=2$, as suggested by this result, a Kolmogorov exponent $\mu=$ $\frac{5}{3}$ is obtained for the choice $\beta=1$. For this choice, $u_{m} \sim x_{m}^{-\frac{1}{2}}$, and correspondingly the circulating velocity associated with the spiral is $u(r) \sim$ $r^{-\frac{1}{2}}$. This slower fall-off than the velocity $\left(\sim r^{-1}\right)$ associated with a point-

\footnotetext{
${ }^{2} \mathrm{R}$. Krasny (private communication) confirms this inference in further computations with desingularisation parameter $\delta=0.03$ and a spiral with 21 turns.
} 


\section{By H.K. Moffatt}

vortex is of course related to the more diffuse distribution of vorticity in the spiral region. The value $\beta=1$ means that the jump in velocity is the same across each discontinuity, i.e. that the strength of the vortex sheet is effectively constant except perhaps in the very central region. The evidence available from Krasny's computations is not conclusive on this point, but is certainly not inconsistent with this conclusion.

\section{Discussion}

Visual observations of turbulent flow, and equally, direct numerical simulations (see for example Kerr 1985), provide ample evidence for the existence of vortex tube structures, frequently exhibiting a spiral cross-sectional structure. The suggestion of this paper, which is a development of the argument advanced in Moffatt (1984, 1990), is that a random distribution of vortices of this kind is all that is needed to provide an inertial range spectrum proportional to $k^{-\frac{5}{3}}$. There is certainly no need to consider a hierarchy of structures on different length-scales, with a cascade of energy from one scale to the next. A single generic structure, which evolves in time due to its internal dynamics, and possibly (as in Lundgren's 1982 model) through additional straining associated with other structures, is sufficient to provide an inertial range spectrum of Kolmogorov type, although for very different reasons from those first advanced by Kolmogorov. This does not of course mean that other physical processes (e.g. vortex break-down) may not be important also in turbulent flow. But it does mean that the search for characteristic vortical structures is well worthwhile, not only to provide a better understanding of the dynamics of the energy-containing eddies, but also in understanding the process of transfer of energy to the small scales where viscous dissipation is operative.

\section{References}

1. Batchelor, G.K. (1967). An Introduction to Fluid Dynamics. Cambridge University Press.

2. Batchelor, G.K. (1969). Computation of the energy spectrum in homogeneous two-dimensional turbulence. Phys. Fluids Suppl. II, 233.

3. Batchelor, G.K. (1982). The Theory of Homogeneous Turbulence (Cambridge Science Classics, CUP).

4. Gilbert, A.D. (1988). Spiral structures and spectra in two-dimensional turbulence, J. Fluid Mech. 193, 475-497. 
Spiral structures in turbulent flow

5. Kerr, R.M. (1985). Higher-order derivative correlations and the alignment of small-scale structures in isotropic numerical turbulence. $J$. Fluid Mech. 153, 31-58.

6. Kraichnan, R.H. (1967). Inertial ranges in two-dimensional turbulence. Phys. Fluids 10, 1417-1423.

7. Krasny, R. (1986). Desingularisation of periodic vortex sheet roll-up, J. Computational Physics 65, 292-313.

8. Lundgren, T.S. (1982). Strained spiral vortex model for turbulent fine structure. Phys. Fluids 25, 2193.

9. Moffatt, H.K. (1984). Simple topological aspects of turbulent vorticity dynamics. In Turbulence and Chaotic Phenomena in Fluids, ed. T. Tatsumi (Elsevier) 223-230.

10. Moffatt, H.K. (1990). Fixed points of turbulent dynamical systems and suppression of nonlinearity. In "Whither Turbulence? - Turbulence at the Crossroads", ed. J.M. Lumley. Lecture Notes in Physics 357 (Springer-Verlag) 250-257.

11. Moore, D.W. (1979). The spontaneous appearance of a singularity in the shape of an evolving vortex sheet. Proc. Roy. Soc. A365, 105.

12. Saffman, P.G. (1971). On the spectrum and decay of random twodimensional vorticity distributions at large Reynolds number. Stud. Appl. Maths 50, 377.

13. Townsend, A.A. (1951). On the fine-scale structure of turbulence. Proc. Roy. Soc. A209, 418. 